\title{
REFORÇO DE PILARETES DE CONCRETO ARMADO EXPOSTOS A ELEVADAS TEMPERATURAS COM A UTILIZAÇÃO DE TECIDO DE FIBRA DE CARBONO
}

\author{
D. B. MARQUES \\ Eng. Civil \\ UFRGS \\ RS, Brasil \\ marques.diegob@gmail.com
}

\author{
M. R. GARCEZ \\ Dr. Eng. Civil \\ UFRGS \\ RS, Brasil \\ Monica.garcez@ufrgs.br
}

\author{
A. G. GRAEFF \\ PhD. Eng. Civil \\ UFRGS \\ RS, Brasil \\ angel.graeff@gmail.com
}

\section{RESUMO}

A exposição de estruturas de concreto armado a elevadas temperaturas resulta na redução de propriedades mecânicas, mudanças na coloração, desplacamentos, esfarelamento superficial e fissuração, podendo limitar ou até mesmo impossibilitar o uso futuro da estrutura. Este trabalho avalia, primeiramente, a perda de capacidade de carga de pilaretes de concreto armado submetidos a elevada temperatura. Posteriormente, é avaliada a possibilidade de recuperação de sua capacidade portante por meio de reforço estrutural com tecido de fibra de carbono. Para as condições de ensaio avaliadas, verificou-se que o patamar de temperatura atingido acarreta perdas consideráveis de capacidade de carga nos pilaretes. Diferentes situações de exposição a elevadas temperaturas e de reforço com tecido de fibra carbono que possibilitariam a recuperação plena da capacidade de carga inicial são apresentadas.

Palavras-chave: reforço, fibra de carbono, pilatetes, elevada temperatura.

\section{ABSTRACT}

Exposition of reinforced concrete structures results in the reduction of mechanical properties, color change, detachment, surface crumbling and cracking, which can limit or even preclude the future use of the structure. This work aims at evaluating load capacity loss in reinforced concrete short columns submitted to elevated temperature. Additionally, this work evaluates the possibility to recover the short columns load capacity through structural strengthening with carbon fiber. The temperature threshold reached during the tests causes significant load capacity losses in the short columns. Different combinations of high temperatures exposition versus reinforcement ratio that could result in the full load capacity recovery are assessed.

Keywords: strengthening, carbon fiber, short columns, high temperature.

\section{INTRODUÇÃO}

A exposição de estruturas de concreto armado a elevadas temperaturas resulta na redução de propriedades mecânicas, mudanças na coloração, desplacamentos, esfarelamento superficial e fissuração, podendo limitar ou até mesmo impossibilitar o uso futuro da estrutura. Estas manifestações patológicas variam de acordo com a temperatura máxima atingida pela estrutura, seu período total de exposição e a velocidade de resfriamento, bem como características próprias da estrutura de concreto armado, tais como materiais constituintes e tipo de elemento estrutural (COSTA \& SILVA, 2002). A redução das propriedades mecânicas do concreto e do aço, ocasionadas pela degradação das estruturas de concreto armado submetidas a altas temperaturas é estimada a partir das propriedades destes materiais na condição ambiente, minoradas por coeficientes redutores em função da temperatura atingida, a fim de avaliar a capacidade de suporte residual da estrutura, como especificado na NBR 15200 (ABNT, 2012).

O sistema de reforço com fibra de carbono envolto em matriz epóxi pode ser utilizado nas mais diversas estruturas, sendo indicado para aplicações em vigas, lajes, paredes, silos, reservatórios, túneis e demais elementos estruturais sujeitos à deterioração, seja para possibilitar o acréscimo de sua capacidade de carga ou para diminuir as flechas. 
Reforços com fibra de carbono podem aumentar a capacidade de carga das estruturas, proporcionando uma mudança de uso das mesmas, corrigir erros de projeto ou de execução, recuperação estrutural face ao tempo de uso ou por algum acidente durante a vida útil da estrutura, como o caso de exposição da estrutura de concreto armado a elevadas temperaturas (SOUZA \& RIPPER, 1998).

No caso de pilares de concreto armado, quando o concreto é comprimido axialmente, o efeito de Poisson induz a ocorrência de deformações radiais que tem como resultante a expansão lateral do concreto. Por meio do confinamento da seção das peças axialmente solicitadas, pode-se impedir a deformação transversal do concreto, oriunda da atuação da carga axial, conseguindo-se, assim, aumentar a resistência das mesmas à compressão, além de propiciar um aumento na ductibilidade do elemento reforçado. O sistema de reforço com fibra de carbono pode ser utilizado no reforço de pilares de concreto solicitados axialmente. Neste caso, a resistência à tração das fibras promove uma pressão de confinamento que pode ser utilizada para aumentar ou recuperar sua capacidade de carga original. (MACHADO, 2006).

Desta forma, este trabalho avalia, primeiramente, a perda de capacidade de carga de pilaretes de concreto armado submetidos a elevada temperatura. Posteriormente, é avaliada a possibilidade de recuperação de sua capacidade portante por meio de reforço estrutural com tecido de fibra de carbono.

\section{PROGRAMA EXPERIMENTAL}

\subsection{Pilatetes de concreto}

A NBR 6118 (ABNT, 2014), determina que a seção transversal de pilares não deve apresentar dimensões menores que $19 \mathrm{~cm}$. Adotou-se então uma seção transversal de $20 \times 20 \mathrm{~cm}$. O comprimento foi limitado pela máxima abertura do forno no qual os pilares foram expostos as elevadas temperaturas, no caso $60 \mathrm{~cm}$. O concreto foi dosado para $25 \mathrm{MPa}$. A armadura compreende quatro barras de aço CA-50 de $10 \mathrm{~mm}$ de diâmetro e comprimento de $54 \mathrm{~cm}$ e estribos $5 \mathrm{~mm}$ de diâmetro e comprimento total de $70 \mathrm{~cm}$ cada, espaçados $11 \mathrm{~cm}$. A espessura do cobrimento de concreto das barras longitudinais, igual a $3 \mathrm{~cm}$, foi executada de acordo com a espessura indicada para a classe de agressividade do local, e garantida com a utilização de espaçadores plásticos do tipo roseta (Figura 1). A Tabela 1 mostra a matriz experimental.

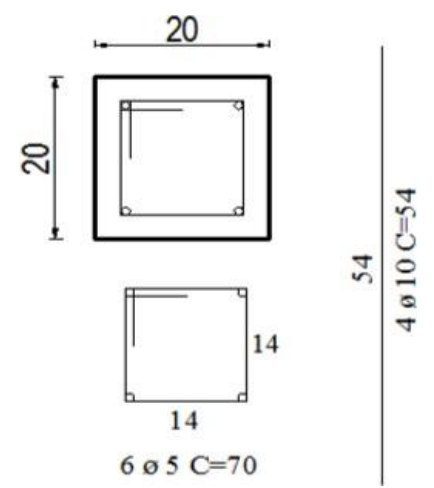

Figura 1: Detalhamento da armadura dos pilatetes.

Tabela 1 - Matriz experimental.

\begin{tabular}{ccccc}
\hline $\begin{array}{c}\text { Pilaretes de } \\
\text { concreto }\end{array}$ & $\begin{array}{c}\text { Grupo de } \\
\text { Concretagem }\end{array}$ & $\begin{array}{c}\text { Elevada } \\
\text { Temperatura }\end{array}$ & $\begin{array}{c}\text { Idade na exposição a } \\
\text { elevada temperatura } \\
\text { (dias) }\end{array}$ & Reforço \\
\hline P1 & Concretagem 1 & Não & - & Não \\
P2 & & Sim & 76 & Não \\
P3 & & Sim & 134 & Sim \\
P4 & Noncretagem 2 & Sim & - & Não \\
P5 & & Sim & 111 & Não \\
P6 & & Não & 111 & Sim \\
P7 & Concretagem 3 & Sim & - & Não \\
P8 & & Sim & 113 & Não \\
P9 & & & 113 & Sim \\
\hline
\end{tabular}




\subsection{Exposição a elevadas temperaturas}

Para cada conjunto de três pilaretes de uma mesma etapa de concretagem, dois foram expostos a elevadas temperaturas em um forno elétrico de alta capacidade de aquecimento, com potência total de $12 \mathrm{~kW} / \mathrm{h}$ e capacidade de aquecimento até $1.340^{\circ} \mathrm{C}$, que simula as condições nas quais os elementos estruturais poderiam ser expostos na condição de um incêndio real.

O procedimento de exposição à elevadas temperaturas consistiu em inserir o pilarete no centro do forno, na posição vertical, e submetê-lo ao período de exposição programado. Para monitorar a temperatura real interna do forno foi utilizado um termopar tipo $\mathrm{K}$, com faixa de medição de temperatura entre $-90^{\circ} \mathrm{C}$ e $1380^{\circ} \mathrm{C}$, conectado a um controlador e programador de temperatura instalado na lateral externa do forno. De acordo com Farias (2015), o forno utilizado para o experimento, mesmo programado na taxa máxima de aquecimento de $100^{\circ} \mathrm{C} / \mathrm{min}$, não atinge os patamares definidos para a curva padrão de incêndio da NBR 5628 (ABNT, 2001). O forno apresentou uma taxa de aquecimento de aproximadamente $17,5^{\circ} \mathrm{C} / \mathrm{min}$ nos 20 minutos iniciais de ensaio, passando para cerca de $2,9^{\circ} \mathrm{C} / \mathrm{min}$ no período seguinte até atingir o patamar desejado de $900^{\circ} \mathrm{C}$. A Figura 2 mostra um comparativo entre a curva de incêndio padrão da norma e a curva de aquecimento do forno utilizado. Após atingida a temperatura desejada $\left(900^{\circ} \mathrm{C} \pm 5 \%\right)$ os elementos foram mantidos neste patamar de temperatura durante 30 minutos, inferior ao tempo mínimo de 120min (TRRF mínimo) para que uma edificação, por exemplo, do tipo residencial (grupo A) classe P5, seja considerada resistente ao fogo, de acordo com a norma NBR 14432 (ABNT, 2001). Quanto ao valor de temperatura, este representa um valor normalmente encontrado na maioria dos incêndios em edificações convencionais. O tempo de exposição 30 minutos foi adotado considerando que como a taxa de aquecimento proporcionada pelo forno é mais baixa que a da curva padrão o pilar permanece exposto as elevadas temperaturas por um período superior durante a fase de aquecimento. Finalizado este período, o forno foi desligado, iniciando a etapa de resfriamento natural dos elementos dentro do próprio forno até ser possível a sua retirada manualmente.

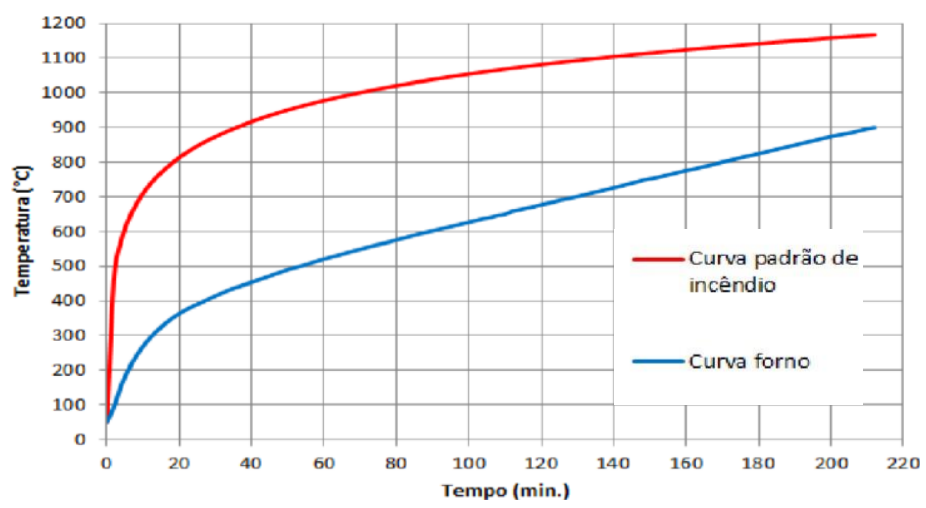

Figura 2: Curva de incêndio padrão versus curva de incêndio do experimento.

\subsection{Dimensionamento do reforço}

As informações apresentadas na Tabela 2 são as condições iniciais para dimensionamento do reforço em função das características dos pilaretes. As propriedades do tecido de fibra de carbono utilizado são descritas na Tabela 3. As cargas inicial e de incêndio são os valores médios obtidos nos ensaios dos pilaretes P1, P4 (excluído P7) e P2, P5, P8, respectivamente.

Tabela 2 - Características dos pilaretes.

\begin{tabular}{lrl}
\hline \multicolumn{3}{c}{ Dados dos Pilaretes } \\
Comprimento $\left(l_{e}\right)$ & 60 & $\mathrm{~cm}$ \\
Lado (b) & 20 & $\mathrm{~cm}$ \\
Lado (h) & 20 & $\mathrm{~cm}$ \\
Área seção $\left(A_{g}\right)$ & 400 & $\mathrm{~cm}^{2}$ \\
Raio de concordância entre lados & 1,5 & $\mathrm{~cm}$ \\
Área de aço armadura principal $\left(A_{s t}\right)$ & 3,1415 & $\mathrm{~cm}^{2}$ \\
Área de aço estribo $\left(A_{\text {est }}\right)$ & 0,1963 & $\mathrm{~cm}^{2}$ \\
Espaçamento estribos $(\mathrm{s})$ & 11 & $\mathrm{~cm}$ \\
$\rho_{g}=A_{\text {st }} / A_{g}$ & 0,007854 & - \\
Carga Inicial $\left(P_{i}\right)$ & 1011,7 & $\mathrm{kN}$ \\
Carga Incêndio $\left(P_{f}\right)$ & 162,26 & $\mathrm{kN}$ \\
\hline
\end{tabular}

Anais do Congresso Brasileiro de Patologia das Construções - CBPAT 2020 
Tabela 3 - Características do tecido de fibra de carbono.

\begin{tabular}{lc}
\hline \multicolumn{2}{c}{ Dados Fibra - Repardur C-Sheet 240 } \\
\hline Módulo Elástico ${ }_{\left(E_{f e}\right)}$ & $240000 \mathrm{MPa}$ \\
Resistência Tração & $3800 \mathrm{MPa}$ \\
Gramatura & $300 \mathrm{~g} / \mathrm{m}^{2}$ \\
Densidade & $1,7 \mathrm{~g} / \mathrm{cm}^{3}$ \\
Extensão de ruptura & $1,55 \%$ \\
Espessura para cálculo $\left(t_{f}\right)$ & $0,0176 \mathrm{~cm}$ \\
Seção transversal teórica & $176 \mathrm{~mm}^{2}$ \\
Fator de redução & 1,2 \\
ft última & $557,3 \mathrm{kN}$ \\
ft última para cálculo $0,6 \% \xi$ & $211 \mathrm{kN}$ \\
\hline
\end{tabular}

Para a determinação da carga de compressão admissível de uma coluna reforçada com fibra de carbono, adotam-se as equações derivadas do American Concrete Institute, descritas em Machado (2006).

Para o cálculo do coeficiente de redução da eficiência da fibra de carbono, foi utilizada a Equação 1, considerando 1,5cm o raio de curvatura (r) entre lados adjacentes (b) e (h).

$$
k_{a}=1-\frac{(b-2 r)^{2}+(h-2 r)^{2}}{3 \cdot b \cdot h \cdot\left(1-\rho_{g}\right)}=0,51452
$$

Através da Equação 2 foi calculada a pressão lateral de confinamento $\left(\rho_{f}\right)$ proporcionada pelo sistema de reforço, em função do número de camadas de fibra (n).

$$
\rho_{f}=\frac{2 . n \cdot t_{f} \cdot(b+h)}{b . h}=0,00352 n
$$

Assim, para seções de colunas retangulares, considerando $f_{f e}=9600 \mathrm{kgf} / \mathrm{cm}^{2}$, a contribuição gerada pelo sistema de reforço é dada pela Equação 3.

$$
f_{l, c f c}=\frac{k_{a} \cdot \rho_{f \cdot} f_{f e}}{2}=8,6933 \cdot n \mathrm{kgf} / \mathrm{cm}^{2}
$$

(Equação 3)

A determinação da contribuição dos estribos existentes, considerando que $f_{\text {s.estrib }}=4200 \mathrm{kgf} / \mathrm{cm}^{2}$, em função das propriedades do aço CA-50 utilizado, é estimada através da Equação 4.

$$
f_{l, e s t r}=\frac{k_{a} \cdot A_{\text {estr }} \cdot f_{s, e s t r i b} \cdot(h+b)}{\mathrm{s} . \mathrm{b} \cdot \mathrm{h}}=3,8572 \mathrm{kgf} / \mathrm{cm}^{2}
$$

A pressão de confinamento se dará pela contribuição do sistema de reforço juntamente com a contribuição dos estribos (Equação 5).

$$
f_{l}=(8,6933 . \mathrm{n}+3,8572) \mathrm{kgf} / \mathrm{cm}^{2}
$$

(Equação 5)

A Tabela 4 mostra a pressão de confinamento estimada considerando diferentes números de camadas de fibra de carbono. $\mathrm{O}$ valor de $f^{\prime}{ }_{c c}$, resistência à compressão majorada pelo confinamento do concreto é fornecido pela Equação 6 , onde $f_{c}^{\prime}{ }_{c} e$ resistência característica à compressão do concreto e $f_{l}^{\prime}$ é a pressão de confinamento.

$$
f^{\prime} c c=f^{\prime} c\left[2,25 \sqrt{1+\frac{7,9 f^{\prime} l}{f^{\prime} c}}-\frac{2 \cdot f^{\prime} l}{f^{\prime} c}-1,25\right]
$$

Para a determinação da carga máxima admissível, usou-se a equação 7, considerando os coeficientes $\phi=0,7$, para casos de compressão axial e estribos normais e $\Psi_{f}=0,95$, como coeficiente adicional de redução da resistência que leva em consideração a forma da seção transversal da coluna. Na Tabela 3, a coluna $\phi P_{n}$, expressa os resultados de carga máxima admissível, levando-se em conta os coeficientes de redução $\phi$ e $\Psi_{f}$ em função do tipo de estribo e geometria da estrutura de concreto, respectivamente. Já a coluna $P_{n}$, desconsidera tais coeficientes.

$$
\phi P n=0,80 \phi x\left[0,85 \Psi f^{\prime} c c x(A g-A s t)+f y A s t\right]
$$

(Equação 7)

Dos resultados apresentados na Tabela 1, tem-se que a capacidade de carga média dos pilaretes testemunhos é de $1011,7 \mathrm{kN}$. Observou-se pelo dimensionamento, conforme os resultados apresentados na Tabela 4, que não seria possível recuperar a totalidade desta capacidade de carga inicial, independente do número de camadas de fibra, pois a medida que este número aumenta, o incremento da resistência torna-se menor. Conforme descrito, como a condição de reforço não recuperaria a capacidade de carga inicial dos pilares, independente do número de camadas de fibra aplicadas, optou-se pelo uso de sistema de fibra de carbono considerando duas camadas. 
Tabela 4 - Dimensionamento de reforço de tecido de fibra de carbono para n camadas.

\begin{tabular}{crrrr}
\hline $\begin{array}{c}\mathrm{n} \\
(\text { camadas })\end{array}$ & $\begin{array}{r}\boldsymbol{f}_{\boldsymbol{l}} \\
\left(\mathrm{kgf} / \mathrm{cm}^{2}\right)\end{array}$ & $\begin{array}{r}\boldsymbol{f}_{\boldsymbol{c} \boldsymbol{c}}^{\prime} \\
\left(\mathrm{kgf} / \mathrm{cm}^{2}\right)\end{array}$ & $\begin{array}{r}\emptyset . \boldsymbol{P}_{\boldsymbol{n}} \\
(\mathrm{kN})\end{array}$ & $\begin{array}{r}\boldsymbol{P}_{\boldsymbol{n}} \\
(\mathrm{kN})\end{array}$ \\
\hline 1 & 12,55 & 93,58 & 255,90 & 378,20 \\
2 & 21,24 & 113,68 & 291,96 & 432,43 \\
3 & 29,94 & 127,95 & 317,59 & 470,96 \\
4 & 38,63 & 138,50 & 336,51 & 499,41 \\
5 & 47,32 & 146,38 & 350,65 & 520,67 \\
6 & 56,02 & 152,24 & 361,16 & 536,49 \\
7 & 64,71 & 156,49 & 368,80 & 547,98 \\
8 & 73,40 & 159,44 & 374,09 & 555,93 \\
9 & 82,10 & 161,29 & 377,41 & 560,93 \\
10 & 90,79 & 162,20 & 379,05 & 563,39 \\
\hline
\end{tabular}

\subsection{Execução do reforço}

A execução do reforço seguiu as seguintes etapas: preparação e regularização da superfície, imprimação, aplicação da resina saturante e aplicação da fibra de carbono. Em virtude da perda considerável de resistência do concreto após a exposição às elevadas temperaturas foi necessária a remoção das partes não aderidas e regularização da superfície com aplicação da argamassa cimentícia de reparo rápido (Figura 3).

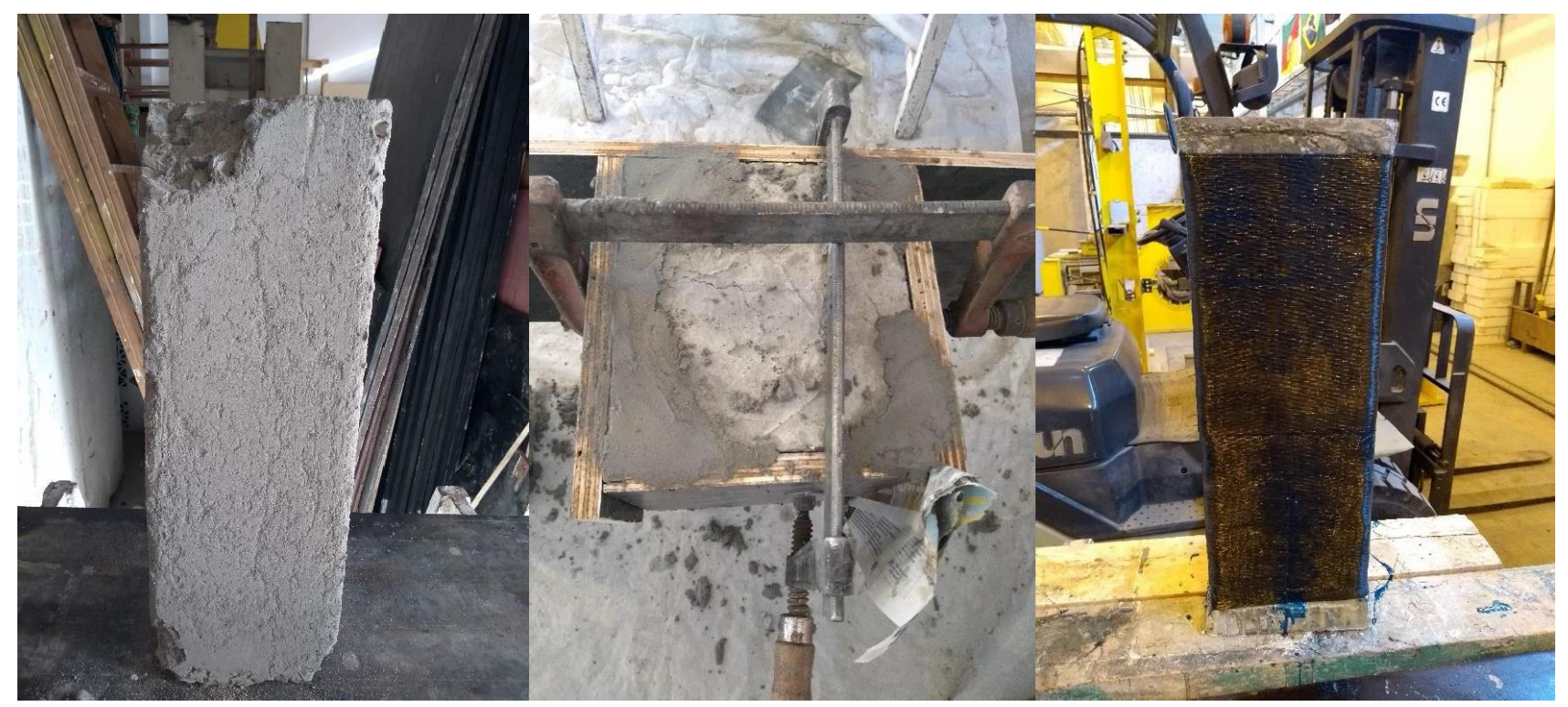

Figura 3: Execução do reforço (a) pilarete danificado após retirada do forno (b) aplicação da argamassa de reparo (c) aplicação da fibra de carbono.

\subsection{Ensaios de compressão axial}

Ensaios de compressão axial foram executados para verificar a capacidade de carga dos pilaretes testemunhos e expostos a elevadas temperaturas, bem como o incremento de carga proporcionado pelo reforço.

\section{ANÁLISE DOS RESULTADOS}

\subsection{Compressão axial}

As Tabelas 5 e 6 apresentam os resultados de capacidade de carga obtidos por meio de ensaio de compressão axial dos pilaretes testemunhos e dos pilaretes expostos a elevada temperatura. 
Tabela 5 - Capacidade de carga dos pilaretes testemunhos.

\begin{tabular}{crr}
\hline Pilarete & $\begin{array}{r}\text { Idade Ensaio } \\
(\text { dias })\end{array}$ & $\begin{array}{r}\text { Carga } \\
(\mathrm{kN})\end{array}$ \\
\hline P1 & 76 & 1004,4 \\
P4 & 100 & 1019,0 \\
P7 & 98 & 822,0 \\
\hline
\end{tabular}

Em função da baixa capacidade de carga do pilarete P2 após ser exposto a elevada temperatura por 120min, decidiu-se reduzir o período de exposição dos demais pilaretes após atingir-se o patamar de $900^{\circ} \mathrm{C}$, conforme descrito no item 2.1 . De fato, como a taxa de aquecimento do forno estava abaixo da considerada na curva de incêndio padrão da norma NBR 5628 (ABNT, 2001), o pilarete ficava exposto a elevada temperatura por um período maior até atingir a temperatura de $900^{\circ} \mathrm{C}$. Após esta alteração, verificou-se, um pequeno incremento na capacidade de carga dos pilaretes P5 e P8 em relação ao P2 da primeira etapa de concretagem.

Tabela 6 - Capacidade de carga dos pilaretes expostos a elevada temperatura.

\begin{tabular}{crrr}
\hline Pilarete & $\begin{array}{r}\text { TRRF } \\
(\mathrm{min})\end{array}$ & $\begin{array}{r}\text { Idade Ensaio } \\
(\text { dias })\end{array}$ & $\begin{array}{r}\text { Carga } \\
(\mathrm{kN})\end{array}$ \\
\hline P2 & $120^{* * *}$ & 76 & 140,0 \\
P5 & 30 & 111 & 193,6 \\
P8 & 30 & 113 & 153,2 \\
\hline
\end{tabular}

A capacidade de carga atingida pelos pilaretes reforçados com tecido de fibra de carbono, está apresentada na Tabela 7. Observa-se que as cargas de ruptura foram superiores à de dimensionamento $\left(\mathrm{P}_{2}=432,43 \mathrm{kN}\right)$, apresentada na Tabela 4 .

Tabela 7 - Capacidade de carga dos pilaretes reforçados com fibra de carbono.

\begin{tabular}{crr}
\hline Pilarete & $\begin{array}{r}\text { Idade Ensaio } \\
(\text { dias })\end{array}$ & $\begin{array}{r}\text { Carga } \\
(\mathrm{kN})\end{array}$ \\
\hline P3 & 134 & 460,0 \\
P6 & 111 & 566,0 \\
P9 & 113 & 455,7 \\
\hline
\end{tabular}

\subsection{Temperatura limite para recuperação da capacidade de carga inicial}

A temperatura máxima que o concreto poderia atingir para conseguir recuperar carga inicial $\mathrm{P}_{\mathrm{i}}=1011,7 \mathrm{kN}$ seria $\phi=$ $300^{\circ} \mathrm{C}$, tendo como base a os coeficientes de redução $\mathrm{K}(\mathrm{c}, \varnothing)=0,85$ da NBR 15200 (ABNT, 2012) e $\mathrm{f}_{\mathrm{ck}}=25 \mathrm{MPa}$. Para esta temperatura o dimensionamento resultaria nos dados apresentados na Tabela 8. A partir dos resultados da Tabela 7 , observa-se que para a temperatura de $300^{\circ} \mathrm{C}$, atingiu-se a capacidade de carga inicial para uma condição de reforço com 2 camadas de fibra, idêntica ao dimensionamento deste programa experimental, desconsiderando novamente os coeficientes de redução. Ainda na Tabela 8 observa-se que para $n=8$, chegou-se a uma capacidade de carga P8 $=1004,72 \mathrm{kN}$, muito próxima da carga inicial e, neste caso, levando-se em consideração todos os coeficientes previstos nas normas de dimensionamento.

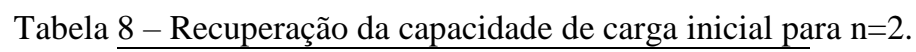

\begin{tabular}{rrrrr}
\hline $\begin{array}{r}\mathrm{n} \\
\text { (camadas) }\end{array}$ & $\begin{array}{r}\boldsymbol{f}_{\boldsymbol{l}} \\
\left(\mathrm{kgf} / \mathrm{cm}^{2}\right)\end{array}$ & $\begin{array}{c}\boldsymbol{f}^{\prime} \boldsymbol{c}\left(\mathrm{kgf} / \mathrm{cm}^{2}\right) \\
\left(\begin{array}{c}\boldsymbol{C}_{\boldsymbol{n}} \\
(\mathrm{kN})\end{array}\right.\end{array}$ & $\begin{array}{r}\boldsymbol{P}_{\boldsymbol{n}} \\
(\mathrm{kN})\end{array}$ \\
\hline 1 & 12,55 & 288,30 & 605,34 & 903,67 \\
2 & 21,24 & 331,53 & 682,93 & 1020,35 \\
3 & 29,94 & 369,50 & 751,07 & 1122,82 \\
4 & 38,63 & 403,38 & 811,86 & 1214,23 \\
5 & 47,32 & 433,95 & 866,73 & 1296,74 \\
6 & 56,02 & 461,79 & 916,69 & 1371,86 \\
7 & 64,71 & 487,31 & 962,49 & 1440,74 \\
8 & 73,40 & 510,84 & 1004,72 & 1504,24 \\
9 & 82,10 & 532,64 & 1043,83 & 1563,05 \\
10 & 90,79 & 552,89 & 1080,18 & 1617,72 \\
\hline
\end{tabular}

A Tabela 9 apresenta os resultados da interpolação da temperatura máxima que teoricamente seria possível recuperar a capacidade de carga inicial integralmente, sem terem sido levados em conta os coeficientes de redução da NBR 15200 (ABNT, 2012). Esta condição ocorreria para uma temperatura máxima de $625^{\circ} \mathrm{C}$ e seriam necessárias 9 camadas de tecido de fibra de carbono de mesmas propriedades das utilizadas neste experimento para se atingir o resultado. 
Tabela 9 - Recuperação da capacidade de carga inicial para $\mathrm{T}=625^{\circ} \mathrm{C}$.

\begin{tabular}{crrrrr}
\hline $\mathrm{n}$ & $\begin{array}{c}\boldsymbol{f}_{\boldsymbol{l}} \\
(\text { camadas })\end{array}$ & $\begin{array}{c}\boldsymbol{f}_{\boldsymbol{c} \boldsymbol{c}}^{\prime} \\
\left(\mathrm{kgf} / \mathrm{cm}^{2}\right)\end{array}$ & $\begin{array}{c}\boldsymbol{\emptyset} \boldsymbol{P}_{\boldsymbol{n}} \\
\left(\mathrm{kgf} / \mathrm{cm}^{2}\right)\end{array}$ & $\begin{array}{c}\boldsymbol{P}_{\boldsymbol{n}} \\
(\mathrm{kN})\end{array}$ & $(\mathrm{kN})$ \\
\hline 1 & 12,55 & 170,96 & 394,76 & 587,01 \\
2 & 21,24 & 204,71 & 455,34 & 678,10 \\
3 & 29,94 & 232,30 & 504,85 & 752,56 \\
4 & 38,63 & 255,53 & 546,53 & 815,24 \\
5 & 47,32 & 275,46 & 582,31 & 869,04 \\
6 & 56,02 & 292,79 & 613,41 & 915,81 \\
7 & 64,71 & 308,00 & 640,70 & 956,84 \\
8 & 73,40 & 321,43 & 664,80 & 993,08 \\
9 & 82,10 & 333,35 & 686,18 & 1025,24 \\
10 & 90,79 & 343,95 & 705,22 & 1053,86 \\
\hline
\end{tabular}

\subsection{Cálculo teórico para a temperatura de $900{ }^{\circ} \mathrm{C}$}

Para $\phi=900^{\circ} \mathrm{C}$, considerando o coeficiente de redução $\mathrm{K}(\mathrm{c}, \emptyset)=0,08$ da NBR 15200 (ABNT, 2012) e $\mathrm{f}_{\text {ck }}=25 \mathrm{MPa}$ a resistência residual do concreto seria $2 \mathrm{MPa}$. A Tabela 10 apresenta uma comparação das capacidades de carga admissíveis, para a redução de resistência do concreto calculada com $\mathrm{K}(\mathrm{c}, \emptyset)=0,08$ e a perda identificada nos experimentos. Os fatores de redução para os estribos e geometria do elemento de concreto armado foram desconsiderados neste caso. A coluna identificada como $\mathrm{P}_{\mathrm{n}}$ experimental apresenta os resultados obtidos através dos experimentos, enquanto a coluna $\mathrm{P}_{\mathrm{n}}$ teórico apresenta os resultados considerando-se a minoração referente à perda de resistência do concreto para uma temperatura de $900^{\circ} \mathrm{C}$. Os dados da Tabela 10 mostram que que os resultados teóricos para a temperatura de $900^{\circ} \mathrm{C}$ ficaram abaixo dos resultados observados através do procedimento experimental realizado.

Tabela 10 - Comparação dos resultados teóricos e experimentais.

\begin{tabular}{|c|c|c|c|c|}
\hline $\begin{array}{r}n \\
\text { (camadas) }\end{array}$ & $\begin{array}{r}f_{l} \\
\left(\mathrm{kgf} / \mathrm{cm}^{2}\right)\end{array}$ & $\begin{array}{r}\boldsymbol{f}_{\boldsymbol{c c}}^{\prime} \\
\left(\mathrm{kgf} / \mathrm{cm}^{2}\right)\end{array}$ & $\begin{array}{r}\boldsymbol{P}_{n} \text { teórico } \\
(\mathrm{kN})\end{array}$ & $\boldsymbol{P}_{\boldsymbol{n}}$ experimental \\
\hline 1 & 12,55 & 59,73 & 286,86 & 378,20 \\
\hline 2 & 21,24 & 70,42 & 315,69 & 432,43 \\
\hline 3 & 29,94 & 76,28 & 331,51 & 470,96 \\
\hline 4 & 38,63 & 79,19 & 339,37 & 499,41 \\
\hline 5 & 47,32 & 80,05 & 341,68 & 520,67 \\
\hline 6 & 56,02 & 79,37 & 339,86 & 536,49 \\
\hline 7 & 64,71 & 77,50 & 334,79 & 547,98 \\
\hline 8 & 73,40 & 74,65 & 327,10 & 555,93 \\
\hline 9 & 82,10 & 70,98 & 317,22 & 560,93 \\
\hline 10 & 90,79 & 66,63 & 305,48 & 563,39 \\
\hline
\end{tabular}

\section{CONSIDERAÇÕES FINAIS}

A partir dos dados obtidos da etapa experimental, conclui-se que a condição na qual os pilaretes foram expostos promoveu um grau de deterioração na estrutura de concreto armado muito elevado. Mesmo com a redução do período de exposição após atingir-se a temperatura máxima de $900^{\circ} \mathrm{C}$ de 120 para 30 minutos, para os pilares da $2^{\mathrm{a}}$ e $3^{\mathrm{a}}$ etapa de concretagem, não houve alteração considerável na capacidade de carga verificada nos pilaretes. A perda de capacidade de carga dos pilaretes expostos a elevadas temperaturas, em relação aos testemunhos, foi em média $84 \%$. Assim, conclui-se que para a condição de ensaio avaliado e da forma de exposição da estrutura a elevadas temperaturas, este patamar de temperatura torna inviável a possibilidade de reforço com tecido de fibra de carbono para recuperação plena da capacidade de carga da estrutura.

Com relação aos resultados obtidos para os pilaretes reforçados com tecido de fibra de carbono, conclui-se que estes atingiram os resultados esperados. Considerando que o cálculo de dimensionamento de reforço realizado, para um sistema com 2 camadas de fibra de carbono previa a capacidade de carga $P_{2}=432,43 \mathrm{kN}$, desconsiderando os coeficientes de redução e que a partir da atividade experimental, obteve-se $P_{\text {med }}=493,93 \mathrm{kN}$, para os três pilaretes reforçados, pode-se 
concluir que o procedimento de cálculo utilizado para o dimensionamento do reforço mostra-se coerente com o resultado alcançado.

Os resultados de temperatura limite à qual uma estrutura de concreto armado poderia ser exposta para recuperação plena de sua capacidade de carga inicial, para as mesmas condições de ensaio efetuadas nesta atividade experimental, irão variar de acordo com o número de camadas de reforço com fibra de carbono possíveis. Vale ressaltar que o uso de uma elevada quantidade de camadas além de dificultar o processo executivo, inviabiliza financeiramente seu uso, tendo em vista o alto custo/m² deste tipo de solução.

\section{REFERÊNCIAS}

ASSOCIAÇÃO BRASILEIRA DE NORMAS TÉCNICAS. NBR 5628: Componentes construtivos estruturais determinação da resistência ao fogo. Rio de Janeiro, 2001.

ASSOCIAÇÃO BRASILEIRA DE NORMAS TÉCNICAS. NBR 6118: Projeto de estruturas de concreto procedimento. Rio de Janeiro, 2014.

ASSOCIAÇÃO BRASILEIRA DE NORMAS TÉCNICAS. NBR 14432: exigências de resistência ao fogo de elementos construtivos de edificações - procedimento. Rio de Janeiro, 2001.

ASSOCIAÇÃO BRASILEIRA DE NORMAS TÉCNICAS. NBR 15200: Projeto de estruturas de concreto em situação de incêndio. Rio de Janeiro, 2012.

COSTA, N. C.; SILVA, V. P. Estruturas de concreto armado em situação de incêndio. In: JORNADAS SUL AMERICANAS DE ENGENHARIA ESTRUTURAL, 2002, Brasília. Anais... Brasília, UnB, 2002.

FARIAS, P. V. Pilares de concreto armado com armaduras corroídas submetidas a altas temperaturas: capacidade portante pós deterioração. 2015. 97 f. Trabalho de Diplomação (Graduação em Engenharia Civil) - Departamento de Engenharia Civil, Universidade Federal do Rio Grande do Sul, Porto Alegre, 2015.

MACHADO, A. P. Reforço de estruturas de concreto armado com fibras de carbono. São Paulo: Pini, 2002. SOUZA, V. C. M.; RIPPER, T. Patologia, recuperação e reforço de estruturas de concreto. 1. ed. São Paulo: Pini, 1998. 